\title{
Effects of OK-432 Activation on the Sialidase Activities of Rat Peritoneal Macrophages
}

\author{
Junji Sagawa, ${ }^{*} \dagger$ Taeko Miyagi* and Shigeru Tsuiki* \\ *Biochemistry Laboratory, the Research Institute for \\ Tuberculosis and Cancer, Tohoku University, and the \\ Second Department of Surgery, Tohoku University School \\ of Medicine, Sendai 980
}

Sagawa, J., Mryagi, T. and Tsuiki, S. Effects of OK-432 Activation on the Sialidase Activities of Rat Peritoneal Macrophages. Tohoku J. Exp. Med., 1990, 160 (2), 149-156 — Intraperitoneal treatment of rat peritoneal macrophages with OK-432 results in more than 9-fold increase in the activity of ganglioside sialidase, which seems to coincide with the appearance of a cell surface antigen, asialo-GM1. The results of subsequent studies suggest that the ganglioside sialidase is located in the plasma membrane, where the enzyme may be responsible for the formation of asialo-GM1 from GM1. In the macrophages activated with OK-432, sialidase activity toward 4-methylumbelliferyl-N-acetylneuraminic acid (4MU-NeuAc) is also increased. It appears that the $4 \mathrm{MU}-\mathrm{NeuAc}$ sialidase is intralysosomal and is increased together with other acid hydrolases present in the lysosomes.

sialidase ; macrophage; OK-432 activation; asialo GM1

Macrophages conduct processing of antigens and release immune response regulatory factors collectively referred to as monokines. Macrophages therefore play an essential role in the development of cell-mediated immunity. Macrophages are also known to be activated with bacterial immunostimulants such as BCG, Corynebacterium paruvm and Streptococcus pyogenes thereby exhibiting a tumoricidal effect (Poplach et al. 1976; Ruco and Meltzer 1977; Cohn 1978; Kornovsky and Lazdins 1978; North 1978). The activation brings about morphological and functional alterations including those in the activities of lysosomal (Cohn and Benson 1965; Saito and Suter 1965; Michl et al. 1977), membraneassociated (Edelson et al. 1975 ; Edelson and Cohn 1976 ; Edelson and Erbs 1978; Morahan et al. 1980) and other enzymes (Gordon et al. 1974 ; Werb and Gordon 1975). In addition, the activation of peritoneal macrophages of the mouse was found to be accompanied by the appearance of a cell surface antigen, asialo-GM1 (Akagawa and Tokunaga 1982). Asialo-GM1 was also detected on the cell surface of peritoneal exudate cells of the rat (Momoi et al. 1980).

Received December 14, 1989 ; revision accepted for publication January 23, 1990. Abbreviation used : 4MU-NeuAc, 4-methylumbelliferyl-N-acetylneuraminic acid. 
We have previously characterized three types of sialidase (EC 3.2.1.18) in rat liver: they were designated the intralysosomal, cytosolic and plasma membrane sialidases according to their major subcellular location (Miyagi and Tsuiki 1984, 1985, 1986). In attempts to gain further insights into the roles of these sialidases, we have recently studied how the sialidase activities of peritoneal macrophages of the rat are affected when these cells are activated with OK-432 (Picibanil, Chugai, Tokyo). OK-432 is Su strain of Streptococcus pyogenes that has been inactivated with penicillin G (Okamoto et al. 1967), and its antitumor effect has been demonstrated both experimentally (Okamoto et al. 1967; Matsubara et al. 1979 ; Hojo and Hashimoto 1981) and clinically (Uchida and Hoshino 1980a, b). The results obtained so far are such that OK-432 induces a characteristic timedependent alteration in the activity of plasma membrane sialidase, which may be associated with the appearance of asialo-GM1.

\section{Materials and Methods}

Materials. Ganglioside GM3, ${ }^{14} \mathrm{C}$-labeled in the NeuAc moiety, was prepared as described by Miyagi and Tsuiki (1986). 4MU-NeuAc was obtained from Nakarai (Tokyo) and rabbit anti-asialo-GM1 serum and fluorescence (FITC)-conjugated goat anti-rabbit IgG from Wako Co. (Tokyo).

Treatment with OK-432. OK-432 was generously provided by Dr. K. Kumagai, Tohoku University School of Dentistry. Male Wistar rats (150-200 g) were injected intraperitoneally with OK-432, whose amount corresponded to $0.1 \mathrm{mg}$ of the dried bacteria ; 2 to 11 days after the injection, the animals were decapitated.

Preparation of macrophages. After decapitation, the peritoneal cavity of the animals were washed out with Dulbecco's PBS (phosphate-buffered saline, Nissui, Tokyo), and the washings were centrifuged at $4{ }^{\circ} \mathrm{C}$. The peritoneal cells thus collected were resuspended in RPMI-1640 medium (Wako Co.) supplemented with $10 \%$ fetal calf serum and incubated in plastic flasks (Falcon Chemical Co., Oxnard, CA, USA) at $37^{\circ} \mathrm{C}$ for $60 \mathrm{~min}$ in a $\mathrm{CO}_{2}$ incubator. Non-adherent cells were removed by washing three times with cold PBS, and the adherent cells remaining were scrapped off and used as purified macrophages. Peritoneal macrophages from animals not treated with OK- 432 served as control. Using the same procedure, alveolar macrophages were obtained after intratracheal canulation (Holt 1979) and splenic macrophages were prepared from spleen. More than $95 \%$ of the adherent cells obtained were judged to be macrophages from the morphology and esterase staining of the preparation.

Preparation of the particulate fraction. All the preparation procedures described below were carried out at $4^{\circ} \mathrm{C}$. Macrophages were suspended in $2 \mathrm{ml}$ of $0.25 \mathrm{M}$ sucrose $/ \mathrm{mM}$ EDTA and homogenized in a tight glass/Teflon homogenizer with 20 strokes. After centrifugation at $1,000 \times \mathrm{g}$ for $10 \mathrm{~min}$, the supernatant was further centrifuged at $105,000 \times$ $\mathrm{g}$ for $1 \mathrm{hr}$, and the resultant pellet was resuspended in $0.5 \mathrm{ml}$ of $0.25 \mathrm{M}$ sucrose/ $1 \mathrm{mM}$ EDTA. This suspension was used as the particulate fraction. The final supernatant was examined for the cytosolic sialidase.

Sialidase assays. When ganglioside-sialidase activity was being assayed, the reaction mixture contained $10 \mathrm{nmol}$ of $\left[{ }^{14} \mathrm{C}\right] \mathrm{GM} 3,0.1 \mathrm{mg}$ of Triton $\mathrm{X}-100,5 \mu \mathrm{mol}$ of sodium acetate $(\mathrm{pH} 4.5)$ and 300-500 $\mu \mathrm{g}$ of enzyme in a final volume of $0.1 \mathrm{ml}$. After incubation at $37^{\circ} \mathrm{C}$ for 60-120 min, the radioactivity released was determined as described previously (Miyagi et al. 1986). Sialidase activity was also determined with $4 \mathrm{MU}-\mathrm{NeuAc}$ as substrate: the reaction mixture contained $120 \mathrm{nmol}$ of $4 \mathrm{MU}-\mathrm{NeuAc}, 5 \mu \mathrm{mol}$ of sodium acetate $(\mathrm{pH} 4.5)$ and enzyme in $0.2 \mathrm{ml}$. Cytosolic sialidase was assayed under the same conditions except that 5 
$\mu$ mol of sodium cacodylate ( $\mathrm{pH} 7.0$ ) substituted for sodium acetate. After incubation at $37^{\circ} \mathrm{C}$ for 1-3 hr, 4-methylumbelliferone produced was determined spectrofluorometrically (excitation, $365 \mathrm{~nm}$; emission, $449 \mathrm{~nm}$ ).

Other assay methods. Acid phosphatase, 5'-nucleotidase and alkaline phosphatase were assayed as described by Miyagi and Tsuiki (1986). Protein was determined by using a phenol reagent (Lowry et al. 1951).

Immunofluorescence staining for asialo-GM1 antigen. Peritoneal macrophages were isolated by the method of Kumagai et al. (1979) using a fetal calf serum-coated petri dish. Isolated $1 \times 10^{6}$ macrophages were washed with $\mathrm{PBS} / 0.2 \%$ bovine serum albumin, added with $10 \mu \mathrm{l}$ of appropriately diluted rabbit anti-asialo GM1 serum, and the mixture was kept at $4^{\circ} \mathrm{C}$ for $30 \mathrm{~min}$. After three washes with $\mathrm{PBS} / 0.2 \%$ bovine serum albumin, $10 \mu \mathrm{l}$ of diluted fluorescence (FITC)-conjugated goat anti-rabbit IgG was added, and the mixture was kept again at $4^{\circ} \mathrm{C}$ for $30 \mathrm{~min}$. Macrophages were washed again, added with $0.5 \mathrm{ml}$ of sheath solution (PBS $/ 0.25 \%$ formalin) and passed through Nylon Mesh. The percentage of stained cells were then determined by using a Flow Cytometer (FACS can; Beckton Dickinson Inc., Mountain View, CA, USA).

\section{Results}

Peritoneal macrophages that had been activated intraperitoneally with OK-432 0-11 days before sacrifice were homogenized, and their particulate fraction was assayed for sialidase. Since more than a single type of sialidase were supposed to be present in this fraction (Miyagi and Tsuiki 1984, 1986), the assay was made with 4MU-NeuAc and GM3, the preferential substrate for hepatic intralysosomal (Miyagi and Tsuiki 1984) and plasma membrane sialidase (Miyagi and Tsuiki 1986), respectively. Fig. 1 shows the results obtained. The activation with OK-432 caused more than 9-fold increase in the activity toward $4 \mathrm{MU}$ NeuAc within 2 days followed by a rapid return to the control value as early as on Day 5 (Fig. 1A). The sialidase activity toward GM3 was also increased more than 9 -fold but the maximum activity was attained only on Day 3 ; it then declined very gradually so that on Day 5, the activity was still $80 \%$ of the maximum activity (Fig. 1B). Since the activity toward $4 \mathrm{MU}-\mathrm{Neu} A \mathrm{c}$ is more than 1 order of magnitude greater than the activity toward GM3 (Fig. 1), it is quite reasonable to assume that most of the $4 \mathrm{MU}-\mathrm{NeuAc}$-hydrolyzing sialidase do not participate in GM3 hydrolysis.

Fig. 1A also shows that the profile of the sialidase activity toward 4MU$\mathrm{NeuAc}$ is closely similar to that of acid phosphatase, a marker enzyme for the lysosomes, thereby suggesting that the $4 \mathrm{MU}-\mathrm{NeuAc}$ sialidase here may be identical, or at least closely similar, to the intralysosomal sialidase previously characterized in rat liver (Miyagi and Tsuiki 1984). The finding that the enzyme is inactive toward GM3 (see above) is also consistent with this suggestion since hepatic intralysosomal sialidase has been shown not to attack gangliosides (Miyagi and Tsuiki 1984)

In addition to the sialidase activity toward GM3, Fig. 1B shows how the activities of two plasma membrane marker enzymes, 5 '-nucleotidase and alkaline phosphatase, fluctuate upon the OK-432 activation of peritoneal macrophages. 

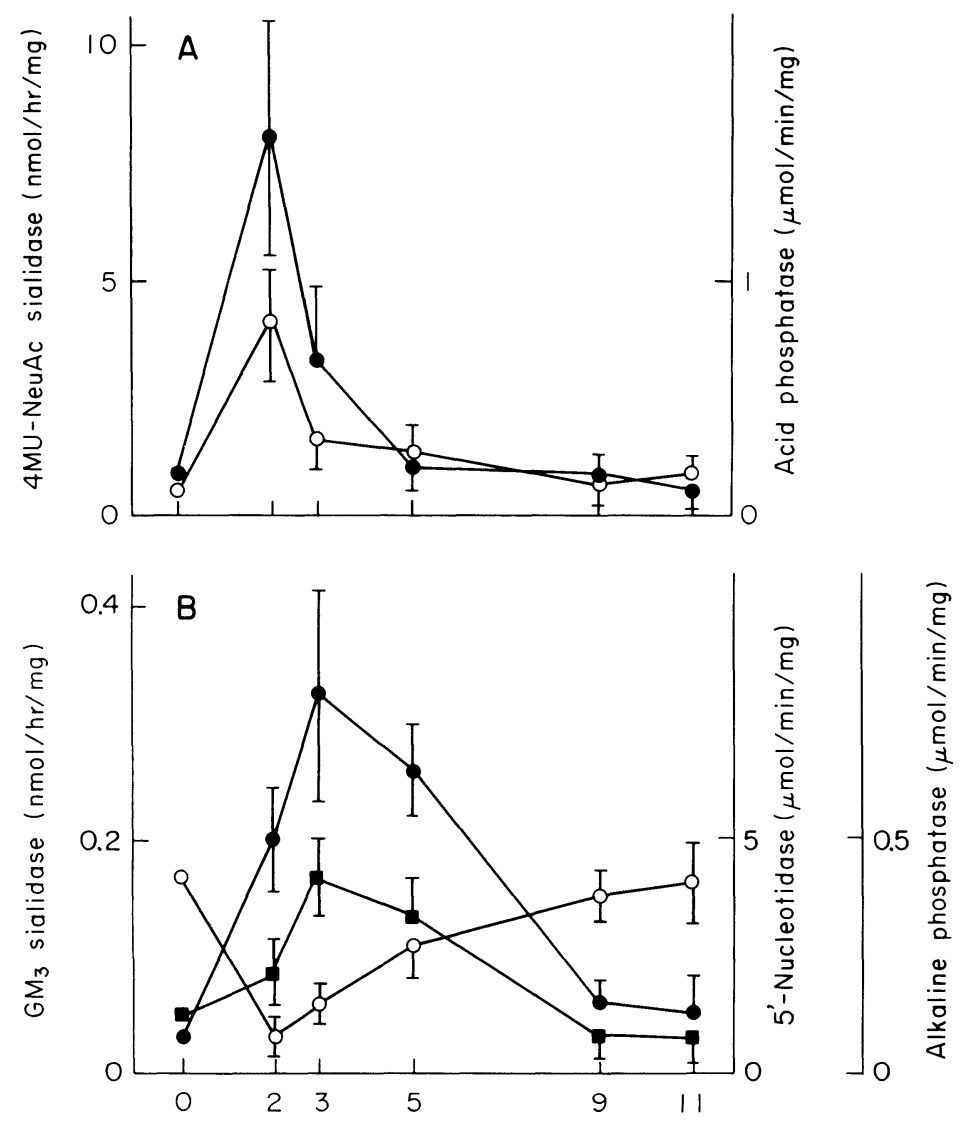

Days after OK-432 intraperitoneal injection

Fig. 1. Effects of OK-432 activation on the sialidase activities of macrophages. Various times after OK- 432 treatment, the particulate fraction was prepared from peritoneal macrophages and assayed for sialidase activities using $4 \mathrm{MU}-$ NeuAc $(\mathrm{A}, \bullet \bullet)$ and GM3 $(\mathrm{B}, \bullet-\bullet)$ as substrate. Other enzymes assayed were acid phosphatase $(\mathrm{A}, \bigcirc-\mathrm{O}), 5^{\prime}$-nucleotidase $(\mathrm{B}, \bigcirc-\mathrm{O})$ and alkaline phosphatase ( $\mathrm{B},-\mathbf{-})$. The values given are means \pm S.D. of three to five experiments.

The profile of alkaline phosphatase is almost in parallel with that of the ganglioside sialidase thereby granting the idea that the latter is also located in the plasma membrane. We have previously demonstrated that in rat liver, the majority of the ganglioside sialidase activity is bound to the plasma membrane (Miyagi and Tsuiki 1986). Although hepatic cytosolic sialidase is also capable of hydrolyzing GM3 (Miyagi and Tsuiki 1985), the enzyme is virtually absent from resident peritoneal macrophages and remains undetectable even after the activation with OK-432 (data not shown). Altogether, these results support the view that the site of the gangliside sialidase shown in Fig. 1B is the plasma membrane (see also Discussion). Although 5'-nucleotidase fluctuates entirely differently 
TABLE 1. The appearance of asialo-GM1 and ganglioside sialidase activity of various types of rat macrophages

\begin{tabular}{|c|c|c|}
\hline Macrophages & $\begin{array}{l}\text { Anti-asialo GM1 } \\
\text { reactive cells }\end{array}$ & $\begin{array}{c}\text { Ganglioside } \\
\text { sialidase } \\
\text { activity }\end{array}$ \\
\hline & $(\%)$ & $(\mathrm{nmol} / \mathrm{hr} / \mathrm{mg})$ \\
\hline \multicolumn{3}{|l|}{ Peritoneal : } \\
\hline Resident & 1.4 & 0.04 \\
\hline 3 days after OK- 432 & 15.1 & $0.33^{\mathrm{a}}$ \\
\hline 5 days after OK- 432 & 22.5 & $0.26^{\mathrm{a}}$ \\
\hline Alveolar & 0.3 & $\mathrm{ND}$ \\
\hline Splenic & 28.5 & 0.96 \\
\hline
\end{tabular}

aTaken from Fig. 1B

ND, not detectable.

from the ganglioside sialidase and alkaline phosphatase, different behaviors of different ectoenzymes are not unusual and have been reported by Edelson and Erbs (1978) and Morahan et al. (1980) for mouse peritoneal macrophages activated in various manners.

Akagawa and Tokunaga (1982) have reported the appearance of a cell surface antigen asialo-GM1 in the activated peritoneal macrophages of the mouse. The OK-432 activation of rat peritoneal macrophages also induces an expression of asialo-GM1. In Table 1, the cells stained with rabbit anti-asialo GM1 serum/ FITC-conjugated goat anti-rabbit IgG serum were only $1.5 \%$ for resident macrophages but were increased to 15.1 and $22.5 \%$ three and five days, respectively, after the OK- 432 treatment. This mode of increase resembles the increase in the ganglioside sialidase (Fig. $1 \mathrm{~B}$ ) rather than the increase in the $4 \mathrm{MU}-\mathrm{Neu}$ Ac sialidase (Fig. 1A). To investigate this point further, the extent of asialo-GM1 expression and the activity of ganglioside sialidase were determined for the alveolar and splenic macrophages of the rat (Table 1). While the alveolar macrophages possess neither asialo-GM1 nor GM3 sialidase activity, they are resonably high in the splenic macrophages. These data are consistent with the hypothesis that the ganglioside sialidase, situated in the plasma membrane, is responsible for the appearance of asialo-GM1 in OK-432-treated peritoneal macrophages.

\section{Discussion}

It is of interest that the activation with OK-432 of rat peritoneal macrophages induces a characteristic time-dependent alteration in their ganglioside sialidase activity (Fig. 1B). Although evidence presented above seems to determine the site of this sialidase to be the plasma membrane, further support to this conclusion has been obtained by our recent unpublished study. In this study, we have produced a rabbit antiserum against the sialidase of rat brain synaptosomal 
membrane (Miyagi et al. 1989); this antibody immunoprecipitated the ganglioside sialidase described above and the sialidases of rat liver plasma membrane and erythrocyte ghost (Sagawa et al. 1989) almost equally thereby supporting the identity of the three sialidases.

The existence of multiple types of sialidase in animal cells is now well established (Miyagi and Tsuiki 1984, 1985, 1986), but their physiological and pathological significance still remains obscure. The present studies have shown that the peritoneal macrophages of the rat express asialo-GM1 subsequently to their activation with OK-432 (Table 1). Since the plasma membrane sialidase of these cells, capable of cleaving GM1 to asialo-GM1, also rises, apparently in concert with the appearance of asialo-GM1, the hypothesis may be put forward that the sialidase is responsible for the cell surface formation of asialo-GM1 from GM1, although the de novo synthesis of asialo-GM1 from lactosylceramide via asialo-GM2 cannot be ignored at present. More thorough studies on the mechanism of asialo-GM1 formation in activated macrophages are now in progress.

Although the 4MU-NeuAc sialidase activity of peritoneal macrophages also rises upon OK-432 activation (Fig. 1A), its participation in the formation of asialo-GM1 is unlikely for the following reasons: (i) the time course of this increase does not coincide with the appearance of asialo-GM1; (ii) the enzyme seems not capable of attacking gangliosides; and (iii) the enzyme would not be accesible to the cell surface gangliosides since it is most probably intralysosomal. It has repeatedly been shown that the activation of macrophages results in increased activities of their lysosomal acid hydrolases (Cohn and Benson 1965; Saito and Suter 1965 ; Michl et al. 1977), and increased upon OK-432 activation.

\section{Acknowledgments}

We wish to express our gratitude to Dr. Katsuo Kumagai (Tohoku University School of Dentistry) for his advice. We also thank Mr. Kimio Konno and Ms. Akiko Kawasaki for their skillful assistance. This work was supported by Grants-in-Aid for Cancer Research from the Ministry of Education, Science and Culture of Japan.

\section{References}

1) Akagawa, K.S. \& Tokunaga, T. (1982) Appearance of a cell surface antigen associated with the activation of peritoneal macrophages in mice. Microbiol. Immunol., 26, 831-842.

2) Cohn, Z.A. (1978) The activation of mononuclear phagocytes: Fact, fancy and future. J. Immunol., 121, 813-816.

3) Cohn, Z.A. \& Benson, B. (1965) The differentiation of mononuclear phagocytosis. J. Exp. Med., 121, 153-169.

4) Edelson, P.J. \& Cohn, Z.A. (1976) 5'-Nucleotidase activity of mouse peritoneal macrophages. J. Exp. Med., 144, 1581-1608.

5) Edelson, P.J. \& Erbs, C. (1978) Plasma membrane localization and metabolism of alkaline phosphodiesterase I in mouse peritoneal macrophages. J. Exp. Med., 147, 77-86.

6) Edelson, P.J., Zmiebel, R. \& Cohn, Z.A. (1975) The pinocytic rate of activated 
macrophages. J. Exp. Med., 142, 1150-1164.

7) Gordon, S., Unkeless, J.C. \& Cohn, Z.A. (1974) Induction of macrophage plasminogen activator by endotoxin stimulation and phagocytosis. J. Exp. Med., 140, 995-1010.

8) Holt, P.G. (1979) Alveolar macrophages. Simple technique for the preparation of high numbers of viable alveolar macrophages from small laboratory animals. $J$. Immunol. Methods, 27, 189-198.

9) Hojo, H. \& Hashimoto, Y. (1981) Cytotoxic cells induced in tumor-bearing rats by a Streptococcus preparation (OK-432). Gann, 72, 692-699.

10) Kornovsky, M.L. \& Lazdins, J.K. (1978) Biochemical criteria for activated macrophages. J. Immunol., 121, 809-813.

11) Kumagai, K., Itoh, K., Hinuma, S. \& Tada, M. (1979) Pretreatment of plastic petri dishes with fetal calf serum. A simple method for macrophage isolation. $J$. Immunol. Methods, 29, 17-25.

12) Lowry, O.H., Rosebrough, N.J., Farr, A.L. \& Randall, R.J. (1951) Protein measurement with the Folin phenol reagent. J. Biol. Chem., 193, 265-275.

13) Matsubara, S., Suzuki, F. \& Ishida, N. (1979) Induction of immune interferon in mice treated with bacterial immuno potentiator, OK-432. Cancer Immunol. Immunother., 6, 41-45.

14) Michl, J., Ohlbaum, D.J. \& Silverstein, S.C. (1977) 2-Deoxyglucose selectively inhibits Fc and complement receptor mediated phagocytosis in mouse peritoneal macrophages. J. Exp. Med., 146, 1648-1662.

15) Miyagi, T. \& Tsuiki, S. (1984) Rat-liver lysosomal sialidase. Solubilization, substrate, specificity and comparison with the cytosolic sialidase. Eur. J. Biochem., 141, $75-81$.

16) Miyagi, T. \& Tsuiki, S. (1985) Purification and characterization of cytosolic sialidase from rat liver. J. Biol. Chem., 260, 6710-6716.

17) Miyagi, T. \& Tsuiki, S. (1986) Evidence for sialidase hydrolyzing ganglioside GM2 and GM1 in rat liver plasma membrane. FEBS Lett., 206, 223-228.

18) Miyagi, T., Sagawa, J., Konno, K. \& Tsuiki, S. (1989) Biochemical and immunological evidence for two distinct sialidases acting on gangliosides : Isolation and characterization of the two sialidases from rat brain. Proceedings 10th International Symposium on Glycoconjugates, edited by N. Sharon, H. Lig, E. Duskin \& I. Kahane, p. 307.

19) Momoi, T., Wiegandt, H., Arndt, R. \& Thiele, H.-G. (1980) Gangliotetraosylceramide, the rat $\mathrm{T}$ lymphocyte-macrophage-associated antigen: Chemical detection and cellular distribution. J. Immunol., 125, 2496-2500.

20) Morahan, P.S., Edelson, P.J. \& Gass, K. (1980) Changes in macrophage ectoenzymes associated with anti-tumor activity. J. Immunol., 125, 1312-1317.

21) North, R.J. (1978) The concept of the activated macrophage. J. Immunol., 121, 806-809.

22) Okamoto, H., Shoin, S., Koshimura, S. \& Shimizu, R. (1967) Studies on the anticancer and streptolysin S-forming abilities of haemolytic streptococci. Jpn. J. Microbiol., 11, 323-336.

23) Poplach, D.G., Sher, N.A. Chaparas, S.D. \& Blaese, R.M. (1976) The effect of Mycobacterium bovis (Bacillus Calmette-Guerin) on macrophage random migration, chemotaxis, and pinocytosis. Cancer Res., 36, 1233-1237.

24) Ruco, L.P. \& Meltzer, M.S. (1977) Macrophage activation for tumor cytotoxicity: Induction of tumoricidal macrophages by PPD in BCG-immune mice. Cellular Immunol., 32, 203-215.

25) Sagawa, J., Miyagi, T. \& Tsuiki, S. (1989) Characterization of the major sialidase of various types of rat blood cells: Their comparison with rat liver sialidases. $J$. Biochem., 107, in press. 
26) Saito, K. \& Suter, E. (1965) Lysosomal and hydrolases in mice infected with BCG. J. Exp. Med., 121, 727-738.

27) Uchida, A. \& Hoshino, T. (1980a) Reduction of suppressor cells in cancer patients treated with OK-432 immunotherapy. Int. J. Cancer, 26, 401-404.

28) Uchida, A. \& Hoshino, T. (1980b) Clinical studies on cell-mediated immunity in patients with malignant disease. Cancer, 45, 476-483.

29) Werb, Z. \& Gordon, S. (1975) Secretion of a specific collagenase by stimulated macrophages. J. Exp. Med., 142, 346-360. 